\title{
Toward Personalizing Alzheimer's Disease Therapy Using an Intelligent Cognitive Control System
}

\author{
Hamdi BEN ABDESSALEM ${ }^{1}$ and Claude FRASSON ${ }^{2}$ \\ Département d'Informatique et de Recherche Opérationnelle \\ Université de Montréal, Canada
}

\begin{abstract}
Subjective cognitive decline is an early state of Alzheimer's Disease which affects almost 10 million people every year. It results from negative emotions such as frustration which are more present than healthy adults. For this reason, our work focuses on relaxing subjective cognitive decline patients using virtual reality environments to improve their memory performance. We proposed in our previous work a neurofeedback approach which adapts the virtual environment to each patient according to their emotions using a Neural Agent. We found that the Neural Agent can adapt the environment to each participant but have limitations. This work is a continuation of our approach in which we propose a Limbic Agent able to monitor the interactions between the Neural Agent and patients' emotional reactions, learn from these interactions, and modify the Neural Agent in order to enhance the adaptation to each patient with an Intelligent Cognitive Control System. Our goal is to create a system able to support the Limbic System which is the main area in charge of controlling emotions and creating memory in the human brain. We used data collected form our previous work to train the Limbic Agent and results showed that the agent is capable of modifying the weight of existing rules, generating new intervention rules, and predicting if they will work or not.
\end{abstract}

Keywords. EEG, Virtual Reality, Limbic System, Intelligent Agent, Neurofeedback, Alzheimer's Disease, Intelligent Cognitive Control System.

\section{Introduction}

Alzheimer's disease (AD) is one of the most crucial diseases of our century affecting millions every year. AD has a direct negative effect on memory and cognitive functions which trigger a negative effect on the emotions. Negative emotions such as anxiety, frustration, and apathy are common in $\mathrm{AD}$ patients which reduce their wellbeing significantly [1]. This disease affects more commonly people aged 65 and older with a vulnerability increasing with age [2]. Alzheimer's disease is progressive, and its earliest phase is characterized by a state of subjective cognitive decline (SCD).

Which makes this disease important is that no cure has yet been found, although, some pharmacological interventions may reduce the symptoms. Considering that AD patients experience negative emotions, non-pharmacological treatments which aim to

\footnotetext{
${ }^{1}$ hamdi.ben.abdessalem@umontreal.ca

2 frasson@iro.umontreal.ca
} 
reduce these negative emotions could be a good alternative to reduce AD symptoms and promotes cognitive performance $[3,4]$.

Virtual Reality (VR) has proven to be efficient in treating certain phobias, for instance, spider's phobia [5], and can be used to calm negative emotions. The immersion provided by VR gives a feeling of safety and encourages imagination. In addition, the patient is isolated from external distraction which increases the effect of the environment presented in VR and reduces the possible negative reactions caused by external factors, electroencephalography (EEG) devices help track the emotional states of AD patients interacting with VR environment.

In a previous work [6], we presented a novel approach which uses VR to relax SCD patients, reduce their negative emotions and improve their cognitive performance. We also proposed to use a Neural Agent which adapts the relaxing VR environment and optimize its effect. We conducted experiments and results showed that the approach effectively reduces negative emotions and improve memory performance. In addition, results showed that the Neural Agent is able to optimize the relaxing environment in order to personalize it for each participant by triggering intervention rules on the environment according to patients' emotions.

We also found that the modification performed by the Neural Agent does not always work as intended, some intervention rules leading to another emotional state not targeted by the rule.

In this work, we aim to verify the following hypotheses: $\mathrm{H} 1$ : it is possible to learn from the interactions between the neural agent and the patient's emotional reactions in order to generate new intervention rules and $\mathrm{H} 2$ : it is possible to predict the impact of the new generated rules on the patient's emotions.

The rest of this paper is organized as follows. In section 2, we present an outline of the related works. Section 3 presents our approach. In section 4 we detail the experiments. Finally, in section 5 we present and discuss the results.

\section{Related Works}

\subsection{Brain Assessment}

Due to its low cost and non-invasiveness, EEG represents a good equipment for brain assessment. In the field of emotion recognition, Liu et al. [7], proposed a multi-level features guided capsule network for multi-channel emotion recognition based on EEG signals. Their framework can identify intrinsic relationship among various EEG channels effectively. Moreover, Zhu et al. [8], analyzed EEG signals in order to explore social emotion perception and emotion classification. They designed a classifier based on convolutional neural network for social emotion classification using EEG. While in the field of psychology, Li et al. [9], proposed a method for quantitative evaluation of people's Big Five personality using EEG. They developed machine learning models in order to predict the user's personality based on their EEG signals while presented to emotional video clips. In the AD and mild cognitive impairment (MCI) detection, Oltu et al., [10] developed a machine learning approach that discriminate $\mathrm{AD}, \mathrm{MCI}$ and healthy control individuals using EEG signals. 


\subsection{Intelligent Agents and Adaptation}

Adaptative systems are often used to personalize experiences for each user. In the field of video games, researchers proposed to adapt a video game in real-time [11]. Using intelligent agents, they track in real-time the player's cognitive and emotional states, then adapt the video game's parameters according to these states. Furthermore, Spencer Rugaber et al. [12] created a model-based technique for self-adaptation in game-playing agents using intelligent agents. Doctor et al. [13] applied intelligent agents in the field of neuroscience. They used adaptation rules to facilitate learned behaviors' tuning and behavioral changes' tracking in the monitoring of dementia patients living in their own homes. Peña et al. [14] used intelligent agents in the field of learning. They adapted their system to learners by using an adaptation technique which focuses on the navigation tools, navigation strategies and the didactic contents.

\subsection{Virtual Reality and Relaxing Environments}

Recently, VR started gaining interest in many fields due to its remarkable advantages. Its main characteristics are immersion, sense of presence, and interactivity [15]. In fact, VR tricks the users' mind and makes them believe they are in a real world and thus promotes their performance [16]. This technology is applied in the field of psychology. Dana et al. [5] used it to as treatments for certain types of phobias. They created virtual environments in order to simulate situations and thus help patients face their fear. Furthermore, Lorenzo G. et al. [17] used VR in order to enhance the emotional skills of children diagnosed with autism spectrum disorders. Moreover, Coyle et al. [18], used it in the cognitive training. In fact, they assessed the efficacy of computerized cognitive training and virtual reality cognitive training programs for individuals living with MCI or dementia.

\section{Our Approach: Intelligent Cognitive Control System}

We propose an Intelligent Cognitive Control System (ICCS), the goal of an ICCS is to detect emotional and cognitive state of the user, alleviate negative emotions and increase positive emotions, using strategies to adapt the virtual environment in order to create the best favorable conditions in order to improve cognitive process. The ICCS is composed of a Savannah VR environment, Neural Agent and Limbic agent. In this section, we detail Savannah VR, Neural Agent, and Limbic Agent which compose our ICCS.

\subsection{Savannah VR}

We developed a Savannah VR environment using Unity 3D software in order to relax SCD patients. This environment provides is a therapeutic experience aiming to improve the negative state of SCD patients. The patients follow an avatar walking through a savannah and speaking in a soft and relaxing voice. Combined with VR techniques, this environment offers an immersive experience to the participants, so they feel like in a real savannah. The environment was developed [19] taking into consideration several parameters able to calm the patients, for instance the dominant color is warm, the animals 
are calm, and their movement is slow. A soothing piano tune is played as music background. Figure 1 illustrates a screen capture of Savannah VR.

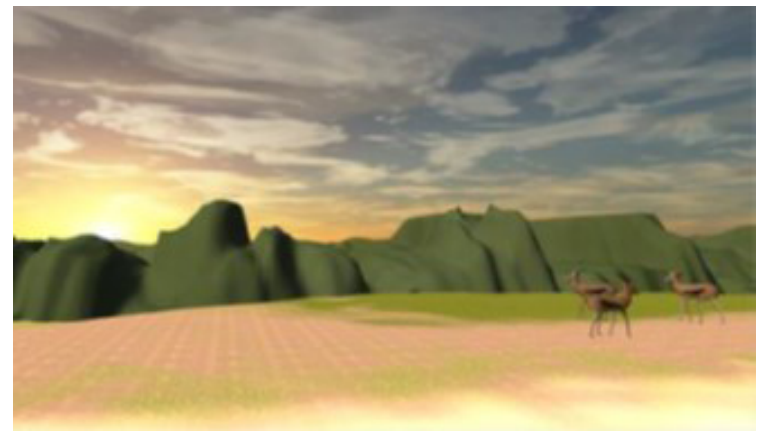

Figure 1. Screenshots of Savannah VR [19]

Participants automatically follow a gazelle that moves along a precise path with breaking points. They are free to look around them by rotating their head in 360 degrees. To avoid nausea caused by movement in virtual reality, users follow the gazelle at low speed. The animal is in front of them to imitate a third-person view that is less likely to cause motion sickness [20].

Our therapeutic approach proposes that the environment's parameters have to be modifiable in real-time. Thus, functions have been implemented that triggers the modification of the environment's parameters.

One of the important parameters to be modifiable is the volume, we must choose the volume carefully; too high volume can cause noise pollution [21]. Another adjustable parameter is the color and intensity of the light because light influence perception and decision-making process [22]. The color can also relieve stress more quickly [23].

As an environment with more trees can also relieve stress more quickly and effectively [24], it is therefore important to control dynamically the number of trees in the environment. The number of animals can also be decreased, and the sky colors can be changed to have a soothing sunset.

\subsection{Neural Agent}

The goal of our work is to optimize and adapt the therapeutic environment to each participant, we propose to use the Neural Agent developed in [11]. This agent is able to modify the parameters of Savannah VR detailed in the previous section based on intervention rules triggering a neurofeedback approach.

This agent possesses a rules base containing multiple intervention rules. The Neural Agent operates by tracking the emotions of the patients and modifies Savannah VR accordingly using intervention rules. Giving the main emotion of the patient, the agent selects a rule from this rules base and modifies the VR environment to adapt it to each patient. The structure of one intervention rule is as following: Rule id, Triggering Emotion, Target Emotion, Action on VR, Weight.

After each intervention, the agent observes the emotional reactions of the patient to check whether or not it does modify the emotional state as desired and updates the weight of the intervention rule.

The Neural Agent is composed of two modules: "Analysis, decision and action" and "Rules base manager" as well as two databases: "Decisions base" and "Rules base". 
Figure 2 illustrates the architecture of the Neural Agent and its interactions with the Emotions module and Savannah VR.

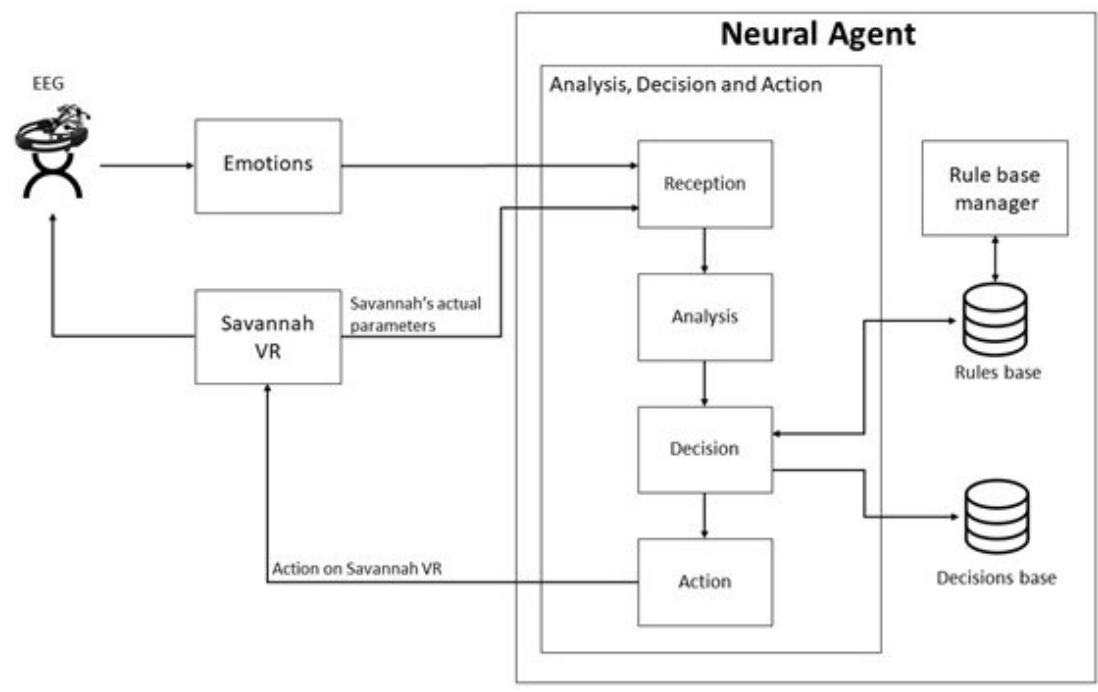

Figure 2. Architecture of the Neural Agent [6].

The "Analysis, Decision and Action" handles the data reception, the data analysis, the decisions to make and the actions to perform on Savannah VR. This module stores the agent's decisions in addition to the user's emotional reaction in the "Decisions base". The structure of a decision in the base is as follows: "Participant Id, Rule Id, Triggering Emotion, Target Emotion, Action on VR, Emotion_1 before Intervention, ..., Emotion_n before Intervention, Emotion_1 after Intervention, ..., Emotion_ $\mathrm{n}$ after Intervention, Worked or Not?"

The "Rules base manager" handles the creation, modification and deletion of the intervention rules and stores them in the "Rules base".

\subsection{Limbic Agent}

The results of our previous work were satisfying but its cognitive capabilities were limited. In order to improve this system, we are interested on how the human brain works and specially the part of the brain which handles the emotions and the memory. We found that the Limbic System is the one which handles the emotions in the brain [25]. Sometimes, the Limbic system cannot regulate the emotions and access to memory. Thus, we propose to create a Limbic Agent which allows our system to assist the human Limbic System by modifying the emotions.

The goal of the Limbic Agent in our system is to observe and analyze the interactions between the Neural Agent and the emotional reactions of the patients, then tries to optimize it and improve it. Thus, it should learn from these interactions, create new intervention rules if needed, and predict its impact on the patient. 
The Limbic Agent is composed of two modules "Observation and Data preparation" and "Learning and prediction" in addition to one database "Knowledge Base". Figure 3 illustrates the architecture of the Limbic Agent and its interactions with the system.

The role of "Observation and Data preparation" is to collect information from all system components through the Neural Agent's Decisions base. This module manages the reception of data and its preparation in the correct format, and sends these data to the "Learning and prediction" module.

The "Learning and prediction" module is composed of four components 'Analysis', 'Learning', 'Rule creation' and 'Prediction'. The role of 'Analysis' component is to receive structured data, analyze which rule works and which rule did not work according to multiple executions of the same rule, analyze the patient's emotional reaction, and change the weight of rules if needed. Then it transmits the data to the 'Learning' and 'Rule Creation' component.

The role of 'Learning' component is to learn from the execution of the intervention rules and the resulting emotional reaction. It creates a prediction model and transmit it to the 'Prediction' component. It stores the information in the 'Knowledge base' so more information it gets, more the knowledge base growth and more its model will be precise.

The 'Rule creation' component role is to analyze the different emotional impacts of each rule. It does not check if the rule worked as expected or not (this is the role of 'Analysis component'), however, it verifies if the multiple executions of one rule generates a different emotional reaction. Then, a new intervention rule is generated and sent to the 'Prediction' component.

The role of the 'Prediction' component is to predict the emotional impact of a new intervention rule just created by the 'Rule Creation' component using the learning model created by the 'Learning' component. Then, it inserts the new intervention rule in the Neural Agent's rules base with the predicted weight so it can be used in the next iteration.

The Limbic Agent is a cognitive agent since it could perform complex tasks autonomously by analyzing the interactions between the Neural Agent and the emotional reactions of the patients, learning from these interactions, modifying existing intervention rules, creating new ones, and finally predicting their impact on a patient.

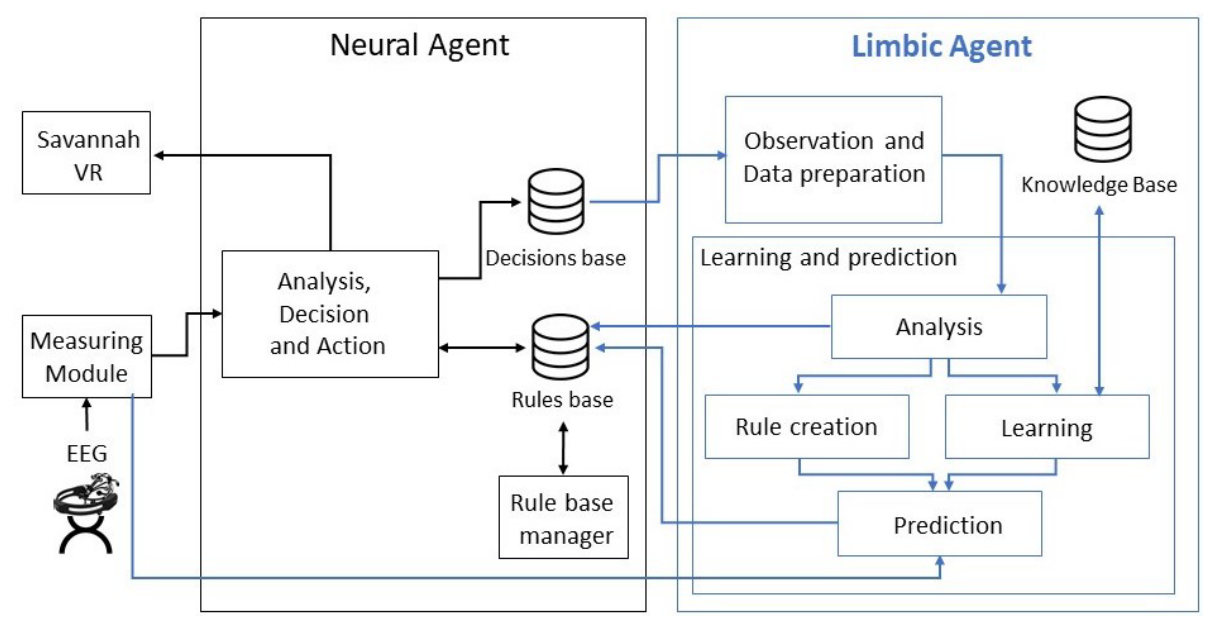

Figure 3. Architecture of the Limbic Agent and its interaction with the system. 


\section{Experiments}

In our previous work [6] we aimed to analyze the impact of the Savannah VR on the memory and attention performances, and for that we created 6 attention and memory exercises. Exercises 1, 2 and 3 are attention tests and exercises 4, 5 and 6 are memory tests.

- Exercise 1: a series of numbers are presented vocally to the participants and they are asked to repeat them in the order of presentation using a numerical pad; then, a second series of numbers are presented vocally and the participants are now asked to report them in the backward order.

- Exercise 2: participants hear a list of letters at a rate of one letter per second and are invited to click the space bar every time they hear the letter " $A$ ".

- Exercise 3: images of different objects are shown for a short period of time. Each image is then replaced by four letters and the participant is asked to select the first letter of the object's name.

- Exercise 4: participants are asked to memorize a series of objects presented visually or verbally. Participants are then presented to a series of objects and are asked to determine whether the object was seen visually, auditorily or never presented.

- Exercise 5: several circles are presented to the participant. A series of these circles is highlighted one by one in order to create a sequence. The participants are asked to memorize and reproduce the same sequence.

- Exercise 6: participants are asked to memorize a set of three pictures for a short period of time. Then, we present four sets and the participants are asked to choose the set which corresponds to the one they saw.

\subsection{Process of the experiments}

We conducted experiments on 19 participants (12 females) with SCD and a mean age = $71(\mathrm{SD}=8.39)$.

The participants took part in two sessions: in the first one, we make sure that they met eligibility criteria to perform the experiments during a pre-experiment session (one hour). Our eligibility criteria were the following:

- Older than aged 60 of age,

- Francophone,

- Normal or correct-to-normal vision,

- Normal hearing,

- Met the Consortium for the Early Identification of Alzheimer's Disease Quebec (CIMA-Q) criteria for SCD:

o Presence of a complaint defined as a positive answer to the following statements: "My memory is not as good as it used to be" "and it worries me."

o MoCA 20-30

o No impairment on the logical memory scale based on the educationadjusted CIMA-Q cut-off scores.

During this first session, participants were provided with oral and written description of the study and were invited to sign a consent form. The session the included the clinical 
tests that are necessary to confirm diagnosis and characterize participants. If the participants were eligible, they were invited to the second session.

In the experimental session, the participants start by filling a pre-session form. Then, we equip them with the Emotiv Epoc+ EEG headset, and they begin to perform attention and memory exercises. When the exercises were completed, we equip them with the Fove VR headset, and they started the savannah relaxing neurofeedback system.

Following the savannah, participants completed other examples of the attention and memory exercises and finished by filling a post-experiment form. Figure 4 details the process of the experiment.

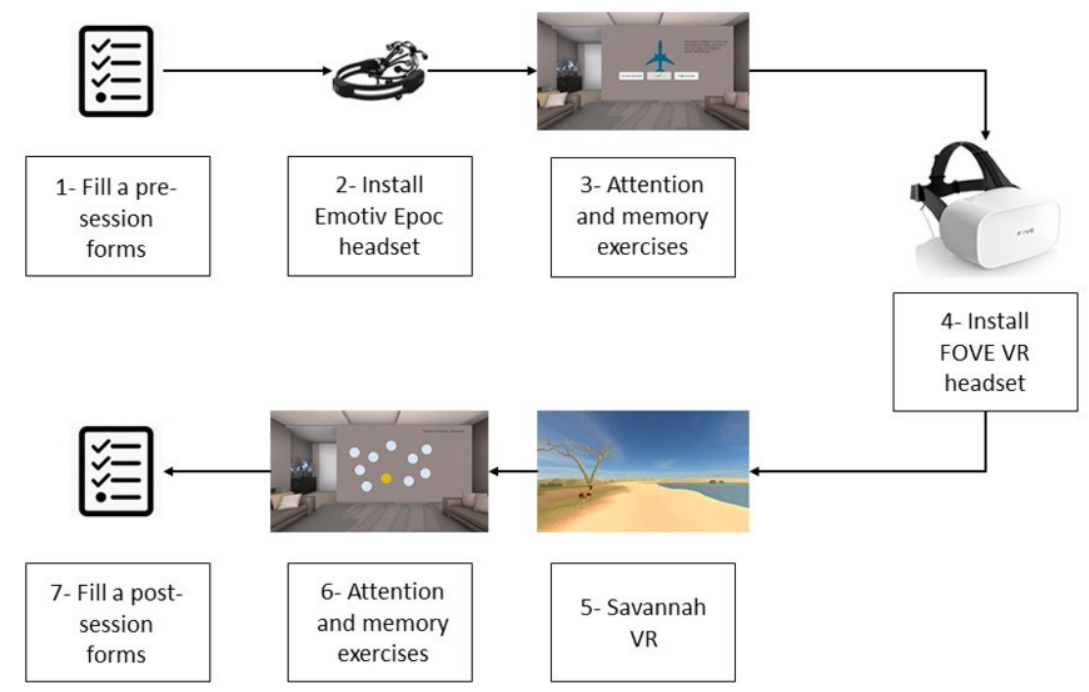

Figure 4. Process of the experiment.

As mentioned in section 3.2, the Neural Agent saves the decisions taken during its interactions with Savannah VR environment in addition to the participants' emotional reactions. We will use the data that the Neural Agent stored during these experiments in order to train the Limbic Agent.

\section{Results and Discussion}

We started by analyzing the interaction between the Neural Agent and the emotional reactions, we noticed that the agent's interventions worked expected 244 on a total of 414 (59\%), however that the interventions did not work 170 times (41\%). Figure 5 illustrates a histogram of the number of worked vs not worked intervention rules. 


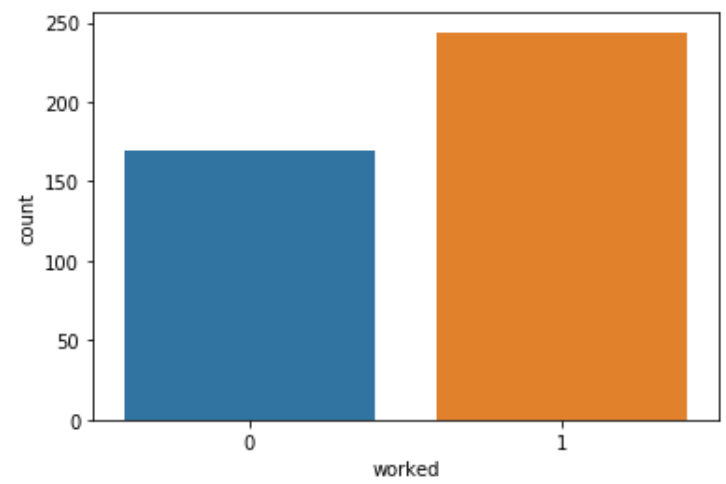

Figure 5. Histogram of the number of worked vs not worked intervention rules.

As we mentioned in section 3.3, the 'Analysis' component of the Limbic Agent analyses the interactions between the Neural Agent and the patients' emotional reaction to each rule and modifies the weight of the existent intervention rules that does not work. For instance, the Limbic Agent found that for participant 4, the intervention rule number 1 was not efficient $75 \%$, so it changes it weight to minimum in order to inhibit it for this particular user. The same rule worked $100 \%$ for participant 18 , so it increases its weight to maximum.

One of the Limbic Agent's objective is to generate new intervention rules considering the interactions between the Neural Agent and the emotional reactions that each rule generates after the intervention. The 'Rule creation' component of the Limbic Agent generated this new rule after analyzing the Neural Agents' Decisions base:

$$
\begin{gathered}
\text { rule_id }=14 \mid \text { triggering_emotion=negative_engagement } \mid \\
\text { target_emotion=positive_excitement } \mid \text { action=light-intensity-incr }
\end{gathered}
$$

This indicates that most of the time, when the patients' engagement was negative and we increase the light intensity, their excitement went up which results to the creation of this intervention rule.

In order to predict the impact of the new intervention rules, we split the Decisions base into $70 \%$ for training and $30 \%$ for testing. We used the 'triggering_Emotion', 'targetting_Emotion', 'action', 'engagement_trend_before', 'frustration_tren_before', 'excitement_trend_before', 'valence_trend_before', 'meditation_trend_before' columns as input features and the model should predict the 'worked' column (labeled 0 or 1 ). We note that the 'Observation and Data preparation' component already normalized the data as follows: it gives a unique integer for each 'trigerring_emotion', 'target_emotion' and action. And for each emotion trend before the intervention rule, it put $\mathbf{1}$ if the emotion goes up, $\mathbf{- 1}$ if it goes down and $\mathbf{0}$ if the trend did not change.

We trained the model that predicts if the intervention rules works or not, and we compared the predicted results of testing data with the already-known labels to analyze the efficiency of the models. Four (4) supervised learning algorithms have been tested in our study, namely: Decision Tree, Random Forest, K-Nearest Neighbors (KNN) and Support Vector Machine (SVM). For Decision tree, we used the Classification and Regression Trees (CART) version [26]. The random forest was set up with 200 estimators. For KNN, the number of k-nearest neighbors was set to 4. Finally. The SVM was set with $\mathrm{C}$ equal to 1.0 and gamma as default.

In order to evaluate the model of each algorithm, we use the precision, recall and F1-score indicators. Precision is the number of true positives divided by the number of 
true positives plus the number of false positives; it reflects the ability of a classification model to identify only the relevant data points. Recall is the number of true positives divided by the number of true positives plus the number of false negatives; it reflects the ability of a model to find all relevant cases within a dataset. F1-Score is a harmonic mean of precision and recall, it reflects how good a model is making a good balance between precision and recall. Table 1 details the results of each algorithm.

Table 1. Classification reports of tested algorithms

\begin{tabular}{rrrrrr}
\hline & & Precision & Recall & F1-score & Support \\
\hline \multirow{3}{*}{ Decision Tree } & 0 & 0.51 & 0.32 & 0.39 & 60 \\
& 1 & 0.53 & 0.72 & 0.61 & 65 \\
& Avg/total & 0.52 & 0.53 & 0.51 & 125 \\
\hline \multirow{3}{*}{ Random Forest } & 0 & 0.55 & 0.28 & 0.37 & 60 \\
& 1 & 0.54 & 0.78 & 0.64 & 65 \\
& Avg/total & 0.54 & 0.54 & 0.51 & 125 \\
\hline \multirow{2}{*}{$\mathrm{KNN}$} & 0 & $\mathbf{0 . 5 8}$ & $\mathbf{0 . 6 2}$ & $\mathbf{0 . 6 0}$ & 60 \\
& 1 & $\mathbf{0 . 6 2}$ & $\mathbf{0 . 5 8}$ & $\mathbf{0 . 6 0}$ & 65 \\
& $\mathrm{Avg} /$ total & $\mathbf{0 . 6 0}$ & $\mathbf{0 . 6 0}$ & $\mathbf{0 . 6 0}$ & 125 \\
\hline \multirow{2}{*}{$\mathrm{SVM}$} & 0 & 0.62 & 0.22 & 0.32 & 60 \\
& 1 & 0.55 & 0.88 & 0.67 & 65 \\
& $\mathrm{Avg} /$ total & 0.58 & 0.56 & 0.50 & 125 \\
\hline
\end{tabular}

Average precision ranged from 0.52 to 0.6 , average recall ranged from 0.53 to 0.6 , and F1-score ranged from 0.5 to 0.6 . However, we note that for all algorithms except KNN, the models miss-predicted too much class 0 which justifies the low recall and F1-score. KNN model is the only one which predicted both classes ( 0 and 1$)$ with an accuracy of $60 \%$ and has an acceptable Recall and F1-score for both classes. Table 2 details the resulting confusion matrix for each tested algorithm.

Table 2. Confusion matrix of tested algorithms

\begin{tabular}{cccc}
\hline & & Predicted 0 & Predicted 1 \\
\hline \multirow{2}{*}{ Decision Tree } & Actual 0 & 19 & 41 \\
& Actual 1 & 18 & 47 \\
\hline \multirow{2}{*}{ Random Forest } & Actual 0 & 17 & 43 \\
& Actual 1 & 14 & 51 \\
\hline \multirow{2}{*}{ KNN } & Actual 0 & 37 & 23 \\
& Actual 1 & 27 & 38 \\
\hline \multirow{2}{*}{ SVM } & Actual 0 & 13 & 47 \\
& Actual 1 & 8 & 57 \\
\hline
\end{tabular}

These results can be improved by performing more experiments to increase the Neural Agents' Decisions base and thus increase the size of training data. The more data the 'Learning' component can have, the more knowledge it can get and the more precision the 'Prediction' component can produce.

\section{Conclusion and Future Work}

We presented in this work an approach aiming to reduce the negative emotions of patients with subjective cognitive decline. We used a Neural Agent which intervenes on a relaxing environment aiming to optimize it and better reduce negative emotions. Experiments were performed and results showed that we can optimize the reduction of negative emotions by controlling the relaxing environment, we proposed an approach 
consisting of an Intelligent Cognitive Control System (ICCS) able to interact with patient through its brain limbic system. This ICCS is composed of the Neural Agent in addition to a Limbic Agent.

The Limbic Agent was created to make the interactions between the system and the human's Limbic System works better. It analyzes the Neural Agent's intervention and the patients' emotional reaction to this intervention, learn from these interactions and makes corrections to the rules base. In addition, it generates new intervention rules that could work better and predicts their impact. Results showed that the Limbic Agent is capable to analyze the interaction and modify the existing rules by increasing and decreasing their weight. Furthermore, results showed that the agent can generate new rules and predict their efficiency using KNN model with a precision of $60 \%$.

Future work consists of making more experiments to collect more data so that we can improve the trained models and get better results. With more data we could use deep learning techniques which could produce better results. Also, we aim to perform multiple sessions experiments with the same persons so that we can see the evolution of the personalization for each person.

\section{Acknowledgments}

We acknowledge NSERC-CRD (National Science and Engineering Research Council Cooperative Research Development), Prompt, and BMU (Beam Me Up) for funding this work.

\section{References}

[1] M. Benoit, I. Dygai, O. Migneco, P. Robert, C. Bertogliati, J. Darcourt, J. Benoliel, V. Aubin-Brunet, D. Pringuey, Behavioral and psychological symptoms in Alzheimer's disease, Dementia and Geriatric Cognitive Disorders. 10 (1999) 511-517.

[2] P.A. Boyle, L. Yu, S.E. Leurgans, R.S. Wilson, R. Brookmeyer, J.A. Schneider, D.A. Bennett, Attributable risk of Alzheimer's dementia attributed to age-related neuropathologies, Annals of Neurology. 85 (2019) 114-124.

[3] H.B. Abdessalem, A. Byrns, M. Cuesta, V. Manera, P. Robert, M.-A. Bruneau, S. Belleville, C. Frasson, Application of Virtual Travel for Alzheimer's Disease, 9th International Conference on Sensor Networks. (2020) 52-60.

[4] A. Byrns, H.B. Abdessalem, M. Cuesta, M.-A. Bruneau, S. Belleville, C. Frasson, Adaptive Music Therapy for Alzheimer's Disease Using Virtual Reality, in: Springer, 2020: pp. 214-219.

[5] D. Horváthová, V. Siládi, Creating virtual environments for phobia treatment, Open Computer Science. 6 (2016) 138-147. https://doi.org/10.1515/comp-2016-0012.

[6] Ben Abdessalem, H., Byrns, A. et Frasson, C. Optimizing Alzheimer's Disease Therapy Using a Neural Intelligent Agent-Based Platform. International Journal of Intelligence Science. (2021). 11(02), 70-96. https://doi.org/10.4236/ijis.2021.112006

[7] Y. Liu, Y. Ding, C. Li, J. Cheng, R. Song, F. Wan, X. Chen, Multi-channel EEG-based emotion recognition via a multi-level features guided capsule network, Computers in Biology and Medicine. 123 (2020) 103927. https://doi.org/10.1016/j.compbiomed.2020.103927.

[8] L. Zhu, C. Su, J. Zhang, G. Cui, A. Cichocki, C. Zhou, J. Li, EEG-based approach for recognizing human social emotion perception, Advanced Engineering Informatics. $46 \quad$ (2020) 101191. https://doi.org/10.1016/j.aei.2020.101191.

[9] W. Li, X. Hu, X. Long, L. Tang, J. Chen, F. Wang, D. Zhang, EEG responses to emotional videos can quantitatively predict big-five personality traits, Neurocomputing. 415 (2020) 368-381. https://doi.org/10.1016/j.neucom.2020.07.123. 
[10] B. Oltu, M.F. Akşahin, S. Kibaroğlu, A novel electroencephalography based approach for Alzheimer's disease and mild cognitive impairment detection, Biomedical Signal Processing and Control. 63 (2021) 102223. https://doi.org/10.1016/j.bspc.2020.102223.

[11] Ben Abdessalem, H. et Frasson, C. Real-time Brain Assessment for Adaptive Virtual Reality Game : A Neurofeedback Approach. Dans C. Frasson et G. Kostopoulos (dir.), Brain Function Assessment in Learning (2017) 133-143. https://doi.org/10.1007/978-3-319-67615-9 12.

[12] S. Rugaber, A.K. Goel, L. Martie, GAIA: A CAD Environment for Model-Based Adaptation of GamePlaying Software Agents, Procedia Computer Science. $16 \quad$ (2013) 29-38. https://doi.org/10.1016/j.procs.2013.01.004.

[13] F. Doctor, R. Iqbal, R.N.G. Naguib, A fuzzy ambient intelligent agents approach for monitoring disease progression of dementia patients, J Ambient Intell Human Comput. 5 (2014) 147-158. https://doi.org/10.1007/s12652-012-0135-x.

[14] C. Peña, J.-L. Marzo, J.D.L. Rosa, Intelligent Agents in a Teaching and Learning Environment on the Web, in: 2002.

[15] K.R. Walsh, S.D. Pawlowski, Virtual Reality: A Technology in Need of IS Research, CAIS. 8 (2002) 296-314. https://doi.org/10.17705/1CAIS.00820.

[16] F. Biocca, M.R. Levy, Communication in the age of virtual reality, 1995.

[17] G. Lorenzo, A. Lledó, J. Pomares, R. Roig, Design and application of an immersive virtual reality system to enhance emotional skills for children with autism spectrum disorders, Computers \& Education. 98 (2016) 192-205. https://doi.org/10.1016/j.compedu.2016.03.018.

[18] H. Coyle, V. Traynor, N. Solowij, Computerized and Virtual Reality Cognitive Training for Individuals at High Risk of Cognitive Decline: Systematic Review of the Literature, The American Journal of Geriatric Psychiatry. 23 (2015) 335-359. https://doi.org/10.1016/j.jagp.2014.04.009.

[19] Dakoure, C., Ben Abdessalem, H., Boukadida, M., Cuesta, M., Bruneau, M.-A., Belleville, S. et Frasson, C. Virtual Savannah: An Effective Therapeutic and Relaxing Treatment for People with Subjective Cognitive Decline. Dans P. Vlamos, P. Bamidis et C. Frasson (dir.), Brain Function Assessment in Learning (2020) 107-112.

[20] A.M. Durko, J.F. Petrick, Travel as Relationship Therapy: Examining the Effect of Vacation Satisfaction Applied to the Investment Model, Journal of Travel Research. 55 (2016) 904-918. https://doi.org/10.1177/0047287515592970.

[21] A. Biamonti, S. Gramegna, B. Imamogullari, A design experience for the enhancement of the quality of life in people with alzheimer's, in: PRT, 2014: pp. 273-284.

[22] K. Schaler, Travel Therapy: Where Do You Need to Go?, Seal Press, 2009.

[23] G.E. Lancioni, N.N. Singh, M.F. O’Reilly, J. Sigafoos, C. Renna, M. Ventrella, K. Pinto, M.G. Minervini, D. Oliva, J. Groeneweg, Supporting daily activities and indoor travel of persons with moderate Alzheimer's disease through standard technology resources, Research in Developmental Disabilities. 34 (2013) 2351-2359.

[24] L.M. Heinrich, E. Gullone, The clinical significance of loneliness: A literature review, Clinical Psychology Review. 26 (2006) 695-718.

[25] J.W. Papez, A PROPOSED MECHANISM OF EMOTION, Arch NeurPsych. 38 (1937) 725. https://doi.org/10.1001/archneurpsyc.1937.02260220069003.

[26] W. Loh, Classification and regression trees, WIREs Data Mining Knowl Discov. 1 (2011) 14-23. https://doi.org/10.1002/widm.8. 